\title{
ABORDAJE DE LOS SISTEMAS DE CONSTRUCCIÓN EN SECO DE TIPO "ENTRAMADO" (STEEL Y BALLOON FRAME) Y LA SUSTENTABILIDAD EDILICIA EN LA ASIGNATURA CONSTRUCCIONES II - FAU - UNNE: CICLOS 2012 Y 2013
}

\author{
Herminia M. ALÍAS / Anabella SCHUSTER / Daniel VEDOYA / Guillermo J. JACOBO \\ Herminia M. Alías / Anabella Schuster - Auxiliar Docente. E-mail: heralias@arq.unne.edu.ar / anabella.schuster@hotmail.com \\ Daniel Vedoya - Profesor Titular. \\ Guillermo J. Jacobo - Profesor Adjunto, Cátedra Construcciones- UNNE. E-mail: Jacobo, Guillermo. \\ Cátedra Construcciones; FAU, UNNE. Área de la Tecnología y la Producción.
}

Palabras Clave:Construcción en seco. Sistemas de entramado.Steelframe. Balloonframe. Keywords: Dry construction, framing systems, steel frame, balloon frame.

\section{RESUMEN}

Se comentan las experiencias en la enseñanza implementadas en la asignatura Construcciones II (4 año de la carrera de Arquitectura). El eje de articulación es un trabajo práctico integrador, donde los alumnos diseñan un sistema constructivo no convencional para resolver diversos programas arquitectónicos. A partir del año 2009, se han incorporado nuevas alternativas entre las que se incluyen la construcción en seco con sistemas de "entramado" (balloon frame y steel frame). Se aborda además la cuestión de la sustentabilidad y la eficiencia energética edilicia desde la etapa de diseño tecnológico. En el ciclo 2012 se trabajó en el diseño de prototipos de viviendas para un barrio privado y en el ciclo 2013 se diseñaron prototipos de cabañas para un emprendimiento inmobiliario turístico.

\section{ABSTRACT}

The experiences in teaching the subject Construcciones II (4th year of the career of Architecture) are commented here. The axis of articulation is an integrating practical work where students design a constructive not conventional system to solve diverse architectural programs. From the year 2009, the practical work has been incorporating new alternatives of dry construction, between which is included the framing systems (baIloon frame and steel frame). The issue of sustainability and energy efficiency building is included from the stage of technological design. In the 2012 cycle, we worked on the design of prototypes for a private residential neighborhood. In the 2013 cycle were designed prototypes for a tourist cabins for an estate tourist development. 


\section{INTRODUCCIÓN}

El objetivo de la asignatura Construcciones // es difundir los conceptos del método industrial de diseño y de producción de elementos constructivos (que serán utilizados posteriormente, totalmente acabados en fábrica), entre un grupo de actores que en un futuro próximo intervendrán en alguno/s o varios de los eslabones de la cadena productiva de la industria de la construcción: los alumnos de Arquitectura, para sentar las bases y fundamentos de la racionalización constructiva, para su implementación de forma permanente en la región NEA y el país. Se considera importante que estos conceptos, y dentro de ellos los inherentes a la construcción en seco, en sus diferentes alternativas, sean comprendidos por los profesionales y técnicos del área, a fin de que pueda entablarse un diálogo (ALÍAS; MORAN; PILAR; SCHUSTER Y VEDOYA, 2010).

En la actualidad, la necesidad de reducir costos y aumentar la productividad hace que los procesos de racionalización y compatibilización constructiva y dimensional vuelvan a ser considerados. En cuanto a la sustentabilidad, los procesos de racionalización y compatibilización constructiva y dimensional, logrados en gran medida al optar por construcción en seco, generan un mejor aprovechamiento de los componentes constructivos $y$, en consecuencia, la optimización del consumo de materias primas, del consumo de energía para la producción de estos componentes y, por último, de los desperdicios de estos componentes en función de los innumerables cortes que sufren en la fase de construcción (GREVEN Y BALDAUF, 2007).

\section{OBJETIVOS Y. FUNDAMENTOS TEÓ- RICO-PEDAGÓGICOS DE LA EXPE- RIENCIA}

El contexto general en que se inserta la cátedra Construcciones II, dentro de la Facultad de Arquitec- tura y Urbanismo de la Universidad Nacional del Nordeste, define una situación problemática en la que se encuentra, en cierta manera, la génesis del objetivo planteado: diversificar e innovar en el trabajo integrador de diseño de un sistema constructivo no convencional desarrollado por los alumnos, incorporando diversidad, tanto de sistemas constructivos para implementar como de problemas arquitectónicos que resolver, fomentando al mismo tiempo el desenvolvimiento de procesos de desarrollo de la creatividad en el avance de las propuestas (ALÍAS, PILAR Y VEDOYA, 2011).

El Plan de Estudios de la carrera de Arquitectura de la UNNE define un perfil profesional según el cual el arquitecto debe poder insertarse y desempeñarse en los nuevos contextos laborales que la sociedad y el mercado demandan. Sin embargo, existe aún cierta percepción de una limitación para actuar en la práctica, por la fuerte carga, durante la formación académica, del bagaje teórico en un currículum de colección, a veces desconectado del contacto con situaciones de la vida práctica, pese a algunos intentos aislados de varias cátedras de la carrera que implementan, desde hace algún tiempo, experiencias alternativas en la enseñanza. Particularmente aquí se presenta el caso de dos de las cuatro grandes comisiones en que se organiza el curso de esta asignatura, que proponen una modalidad de articulación teoría-práctica desarrollada a través de la resolución de un problema de diseño (de un sistema constructivo no convencional - de entramado - industrializado, aplicado a la materialización de un equipamiento de una temática determinada, que varía en cada ciclo lectivo) mediante la estrategia del taller, y a través de una investigación paralela de los condicionantes y determinantes contextuales reales (de mercado, socioeconómicos, tecnológicos y productivos).

Una de las principales situaciones que promovió el proceso de cambio en la asignatura, implementado a partir del año 2009, ha sido la excesiva repetitividad y monotonía (y la falta de creatividad y originalidad subyacentes) verificadas en los tra- 
1. Fig. 1: Steel Frame
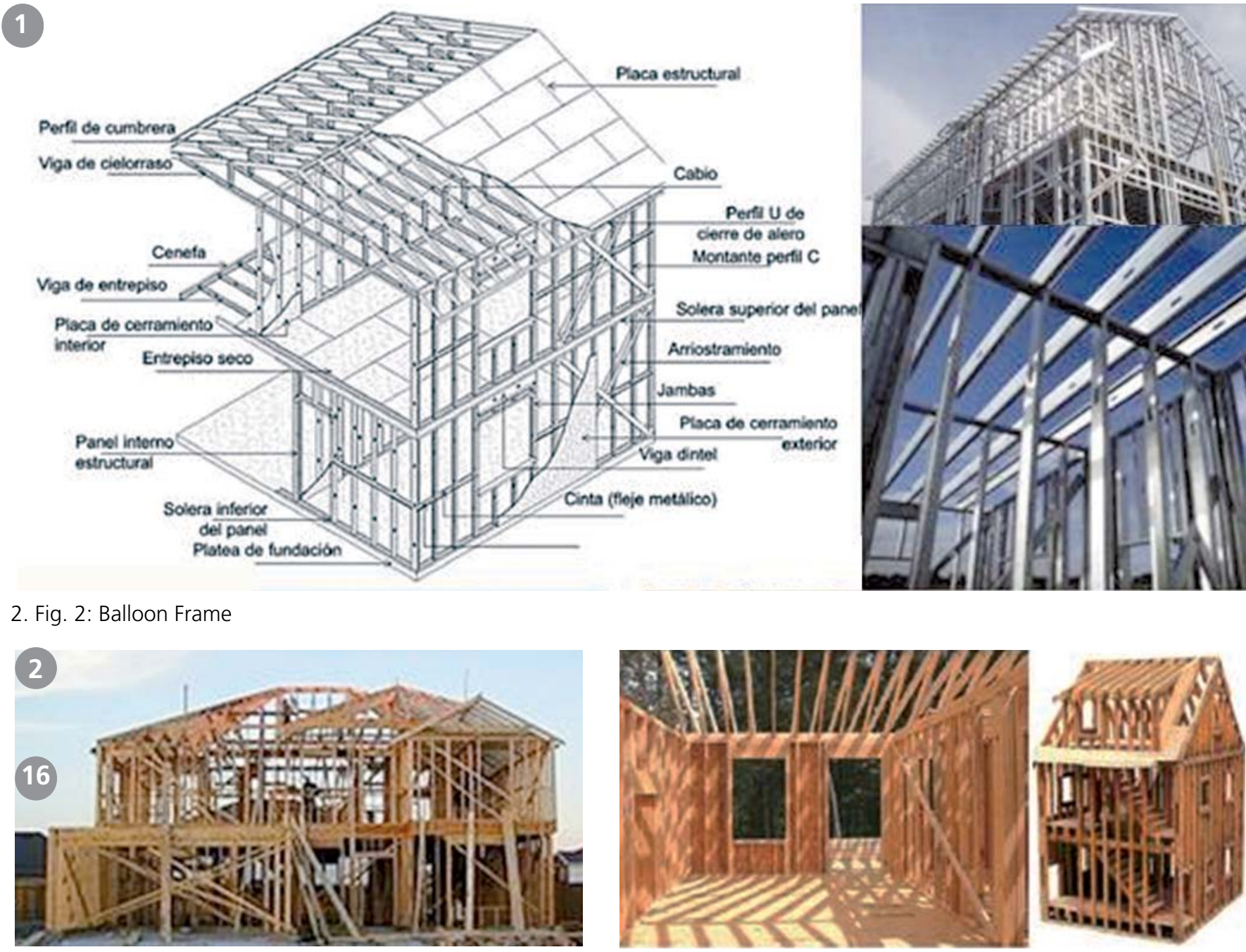

bajos de los alumnos, frente a la repetición año a año del mismo problema constructivo (vivienda unifamiliar) y el uso del mismo sistema constructivo (construcción prefabricada a través de paneles). El alumnado se limitaba a recurrir a la experiencia de los alumnos de los ciclos anteriores y a consultar las producciones de estos, para simplemente diseñar "recreaciones" de lo ya realizado, sin que se verifique un desarrollo de la creatividad mediante aportes originales sustanciales, ni desde el punto de vista técnico ni desde el formal-espacial (ALÍAS, PILAR Y VEDOYA, 2011).

\section{LA CONSTRUCCIÓN EN SECO Y LOS SISTEMAS "DE ENTRAMADO"}

Si bien la construcción en seco resulta el modo de construir habitual en los países desarrollados, en el medio local su implementación resulta poco frecuente, pero su uso se ha incrementado notablemente en los últimos años, por lo que resulta conveniente que los alumnos se interioricen en esta tecnología, ya que resultará de suma utilidad en

su vida profesional. (1. Fig. 1. Steel Frame. Fig. 2. Balloon Frame).

La construcción en seco tiene por objetivo sustituir por elementos secos o prefabricados la mayor cantidad de componentes de una obra, como un hormigón armado, los morteros de cal, cemento, yeso, las mamposterías y todo material que condicione, con su tiempo de fragüe, al rápido avance de la obra. Con la construcción en seco se eliminan de la obra los elementos ya mencionados $y$, fundamentalmente, se reduce el tiempo de convivencia con materiales, equipos y operarios. Sin límite para el diseño, tratándose de un sistema abierto y flexible (flexibilidad para futuros cambios de proyecto), los insumos que componen el sistema son compatibles y posibles de aplicar también en ampliaciones y refacciones de obras existentes. Las combinaciones de materiales posibles son muy amplias, pero básicamente, según cuál sea el material constitutivo básico del entramado (o estructura del sistema), puede hacerse una primera clasificación en dos grandes tipos constructivos "en seco": el "balloon frame" y el "steel frame" (figuras 1 y 
3. Viviendas en 2 plantas diseñadas para ser resueltas mediante el sistema de construcción en seco steel frame.

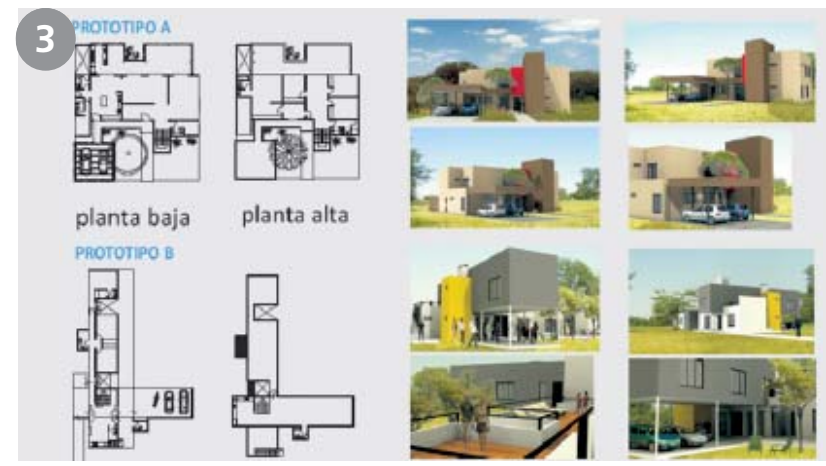

2). Ambos subsistemas de construcción en seco tienen como principal ventaja la gran versatilidad formal de las soluciones, que ha sido aprovechada convenientemente por los alumnos de la asignatura. El balloon frame (estructura de entramado de madera) es un sistema constructivo compuesto por elementos livianos de madera, cuyo concepto básico es la utilización de montantes que tienen la altura total del edificio (generalmente dos plantas), y en donde las vigas del entrepiso están sujetas en forma lateral. El steel frame (estructura de entramado de acero) consiste en la utilización de gran cantidad de elementos estructurales de acero, de sección pequeña, con alta capacidad de resistencia. Las cargas se transmiten por contacto directo de los elementos resistentes, minimizando las piezas sometidas a flexión. Los subsistemas funcionan en conjunto, sin limitaciones de diseño. Es especialmente apto para resistir cargas sísmicas (ALÍAS, SCHUSTER Y VEDOYA, 2010).

La práctica propuesta, con el planteo del problema de diseño del sistema constructivo no convencional, que en el caso que aquí se comenta debe ser realizado por cada equipo de alumnos utilizando alguno de estos sistemas de entramado (metálico o de madera), se basa en el conocimiento de la acción y en el aprender-haciendo, así como en la tutorización antes que la transmisión unidireccional de contenidos del docente al alumno. Se trataría de un "prácticum", "donde el alumno aprende haciendo en una interrelación con tutores y con los otros compañeros en una suerte de aprendizaje experiencial. El tutor acompaña el proceso y puede, si es necesario, enseñar en un sentido tradicional, brindando información o transmitiendo teorías. Pero su función específica es criticar, aconsejar, demostrar y plantear problemas" (FINKELSTEIN Y LUCARELLI, 2003: 10).

\section{MODALIDADES DIDÁCTICAS DE CONSTRUCCIONES}

La modalidad del dictado de la Asignatura Construcciones // es presencial, cuatrimestral, con clases teóricas, teórico-prácticas y de taller, paralelas o conjuntas, según la ocasión, complementadas con
4. Dúplex y de dos dormitorios diseñada para ser resuelta mediante el sistema de construcción en seco balloon frame.

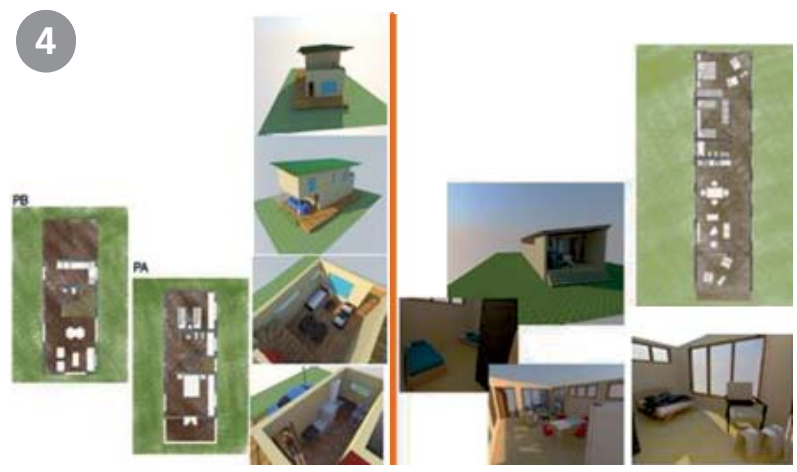

una investigación del mercado y los materiales de construcción regionales. Paralelamente al dictado de clases teóricas, se forman equipos de trabajo para el desarrollo del trabajo de aplicación (en el que se intenta verificar que el alumno compruebe e integre los conceptos trabajados en clase).

En el ciclo 2012 dicho trabajo integrador consistió en el diseño de prototipos de viviendas (Figura 3: viviendas en 2 plantas diseñadas para ser resueltas mediante el sistema de construcción en seco steel frame. Alumnos: Seoane, Sebastián (prototipo de la izquierda) y Seoane Imfeld, Facundo (prototipo de la derecha), ciclo 2012), destinadas a integrar un emprendimiento privado de tipo "barrio cerrado", empleando un sistema de construcción no convencional, con criterios de producción industrial, diseño tecnológico-constructivo sustentable y teniendo en cuenta las condiciones de contexto locales y regionales (aspectos productivos, económicos y sociales). En el ciclo 2013 se hizo lo propio para un emprendimiento inmobiliario de tipo turístico, concretamente diseñando los prototipos (figura 4) de un Complejo de Cabañas (cabañas de 1, 2 y 3 dormitorios -en planta baja y en dúplex-; módulo administrativo y módulo recreativo).

En el interior de cada una de las cuatro grandes comisiones de trabajo (a. construcción prefabricada no integral de pequeñas piezas; b. construcción prefabricada integral -células tridimensionales y reutilización de conteiners- y c. y d. construcción en seco de tipo "entramado": balloon frame y steel frame, respectivamente), los alumnos se organizaron en equipos, de hasta cinco integrantes cada uno, en los que a cada integrante se le asignó un programa arquitectónico diferente. La instancia de diseño del sistema constructivo se planteó en forma grupal, para ser resuelta por el equipo de alumnos, en tanto que la aplicación del sistema a un programa específico se planteó como una instancia individual, al tener cada alumno una función con un programa arquitectónico diferente. (Figura 4: cabaña en dúplex y de dos dormitorios diseñada para ser resuelta mediante el sistema de construcción en seco balloon frame. Alumnos: Urdiales - Morel - Morales - Vañek. Ciclo 2013). La práctica propuesta, a lo largo del problema de 
5. Diseño de la planimetría de la urbanización de carácter privado.

6. Vivienda en dos plantas diseñada para ser resuelta mediante el sistema de construcción en seco steel frame.

5
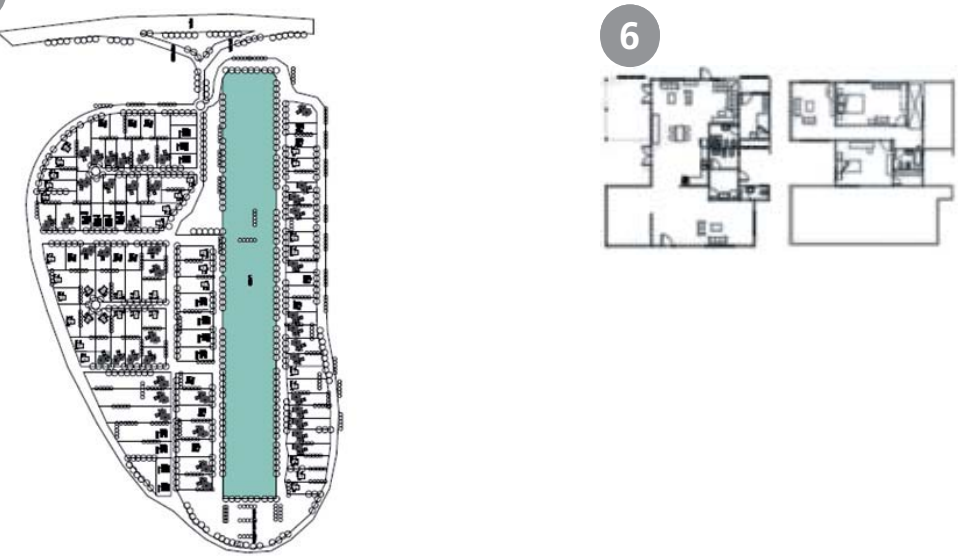

diseño del sistema constructivo no convencional que el equipo de alumnos debe resolver, se basa en el conocimiento de la acción y en el aprenderhaciendo, así como en la tutorización antes que la transmisión unidireccional de contenidos del docente al alumno. El equipo de alumnos debe desarrollar el ejercicio de diseño e investigación en paralelo con el desarrollo de un proceso autogestivo, tratando de adentrarse en el reconocimiento de los principales actores locales o regionales involucrados en la problemática abordada, de modo que los resultados y las diversas instancias del trabajo sean sumamente adaptados a la realidad local del medio y a las necesidades reales y potenciales de los usuarios o posibles comitentes interesados, considerando también herramientas para la comunicación eficaz de sus ideas a dichos posibles interesados (ALÍAS, PILAR Y VEDOYA, 2011).

Ciclo 2012: vivienda para emprendimientos privados El grupo de alumnos debía proponer el diseño de una urbanización con características correspondientes a un emprendimiento privado, implantado en una zona retirada de la ciudad de Resistencia o Corrientes, en lo posible con un espejo de agua (laguna o borde de río), etc. (Figura 5. Planimetría barrio privado. Equipo N. ${ }^{\circ} 45$ : Acosta - Acosta - Maidana Mattar Barrios D'ambra. Ciclo 2012).

El diseño de la planimetría de la urbanización de carácter privado (figura 5) debía ser resuelto, en líneas generales (referentes a cuestiones de implantación, morfológicas y tecnológico-constructivas), en forma grupal en el equipo, en tanto que el programa arquitectónico de la vivienda que integraría la urbanización debía ser interpretado y desarrollado en forma individual por cada uno de los integrantes del equipo de alumnos. Es decir que dicha urbanización constaría de tantos prototipos de vivienda como cantidad de integrantes tuviera el equipo de alumnos. Cada alumno debía proponer el prototipo de una vivienda para integrar dicha urbanización (Fig. 6: vivienda en dos plantas diseñada
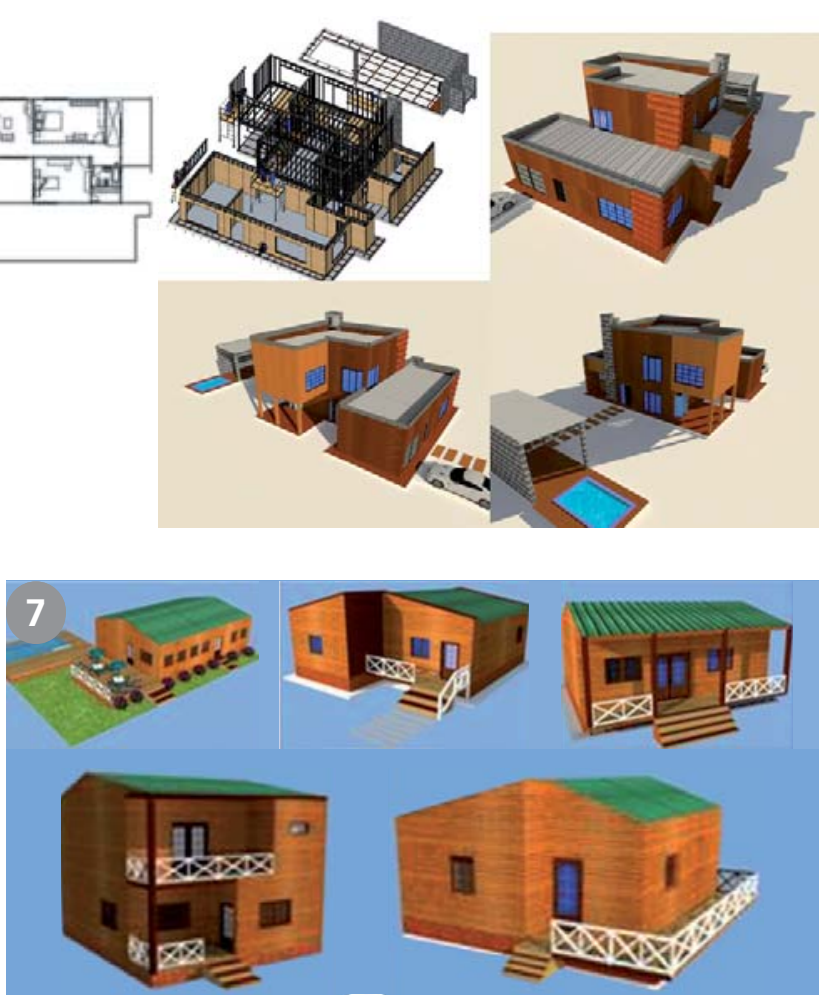

7. Núcleo recreativo, administrativo, cabaña de 1 dormitorio, cabaña de 2 dormitorios, cabaña de 2 dormitorios en dúplex diseñado para ser resuelto mediante el sistema de construcción en seco ballon frame.

para ser resuelta mediante el sistema de construcción en seco steel frame. Alumna: Maidana Mattar, Brenda. Ciclo 2012). La vivienda que diseñar por cada alumno sería de dos plantas o vivienda palafítica, resolviéndose en una superficie mínima de $150 \mathrm{~m}^{2}$ aproximadamente. Debía contar con un living, un toilette, un comedor, una cocina, una cochera, un lavadero con área de tendido, dos dormitorios, un baño, un dormitorio en suite, un quincho con parrilla, pileta, etc. (opcional), una despensa (opcional) y un estudio / biblioteca / sala de juegos (opcional). El prototipo podría ser un diseño propio o la reformulación de un legajo o documentación técnica de una vivienda ya proyectada o construida.

Ciclo 2013: complejo de cabañas para emprendimiento turístico

El grupo de alumnos debía proponer el diseño de un complejo de cabañas implantado en un terreno (de una hectárea o media hectárea) correspondiente a algún punto turístico de la región (Paso de la Patria, Ituzaingó, Isla del Cerrito, Empedrado, Oberá, El Dorado), con la particularidad de que dicho terreno podría ser definido o propuesto por el docente a cargo, o bien podría ser definido o propuesto por el equipo de alumnos, con acuerdo del docente. (Fig. 7: núcleo recreativo, administrativo, cabaña de 1 dormitorio, cabaña de 2 dormitorios, cabaña de 2 dormitorios en dúplex diseñado para ser resuelto mediante el sistema de construcción en seco ballon frame. Grupo N. ${ }^{\circ}$ 17: Alegre, Jessica). Coppola, David - Céspedes, Sonia - Espíndola, Sonia - Romero, Edith. Ciclo 2013 
8. Núcleo húmedo de una vivienda con tendido de cañerías embutidas en cerramientos diseñados para ser resueltos mediante el sistema de construcción en seco balloon frame.
9. Proceso de montaje del Núcleo Administrativo de un Complejo de Cabañas, diseñado para ser materializado mediante un sistema de construcción en seco tipo "steel frame".

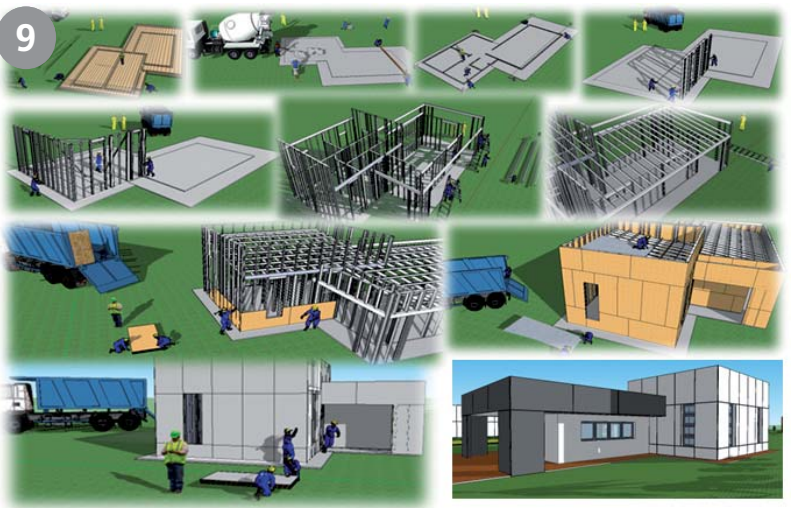

lo cual se encaró el diseño individual del partido del programa que desarrollar por cada integrante del equipo de alumnos, según la función asignada y los criterios de coordinación modular aplicados en forma grupal. Esta etapa incluye el estudio, diseño y desarrollo técnico grupal del sistema de fundación, estructura, techo, piso, cerramientos, así como los encuentros de todos los componentes del sistema constructivo de entramado propuesto.

Como premisa general, y según los criterios de sustentabilidad como condicionantes y determinantes de la propuesta tecnológica, cada equipo de alumnos debió plantear su selección de los patrones modulares de diseño en función de los materiales de trabajo (y sus dimensiones comerciales) que hubieran sido adoptados para la resolución general del sistema constructivo (a efectos de reducir al mínimo los desperdicios por recortes).

Dichos materiales de trabajo para el diseño del sistema constructivo fueron adoptados combinando dos o más de las siguientes opciones generales: placas de cemento; placas de roca de yeso; placas de MDF (medium density fiberboard); placas de OSB (oriented strand board); placas fenólicas o de otras transformaciones industriales de la madera; madera natural y metales en diferentes presentaciones comerciales.

La segunda etapa consistió en las siguientes instancias: 1) el estudio grupal de las aislaciones higrotérmicas propias de la envolvente constructiva de la propuesta (cerramientos laterales exteriores y techo), basados en el cálculo del coeficiente de transmitancia térmica y la verificación del riesgo de condensaciones, según normas IRAM 11601/96, $11605 / 96$ y $11625 / 00 ; 2$ ) el estudio grupal e individual del/los núcleo/s húmedo/s: propuesta de instalaciones de provisión de agua, gas y desagües (figura 8); 3) el estudio grupal e individual de la instalación eléctrica; 4) el planteo grupal e individual del proceso de montaje (figuras 8 y 9), con de- 
10. Panel síntesis del Complejo de Cabañas, diseñado para ser materializado mediante un sistema de construcción en seco tipo "steel frame".

10

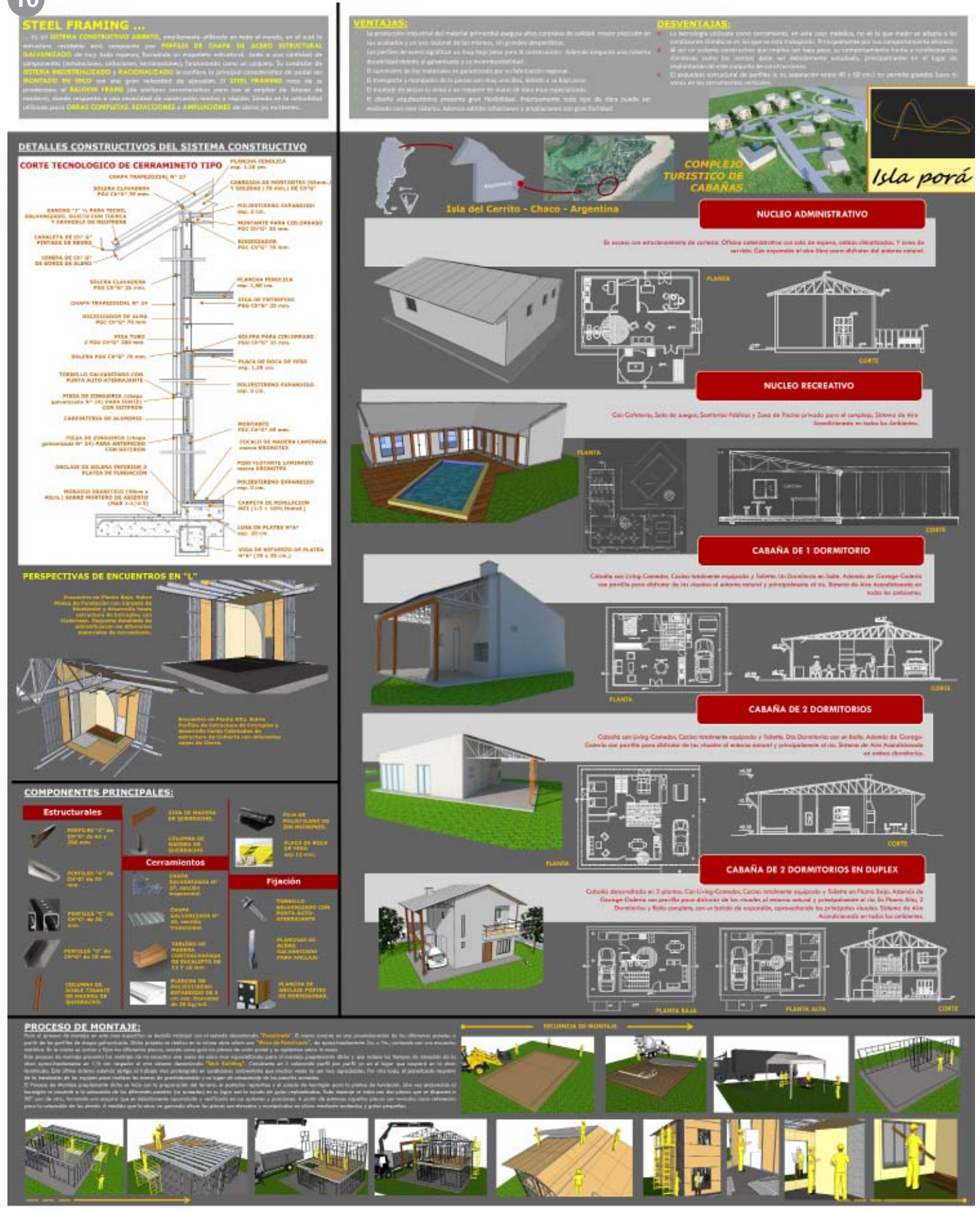

CONSTRUCCIONES II

Docente a cargo: Arq. Alias

STEEL FRAMING

GRUPO No9

CONSTRUCCION EN SECO

faul

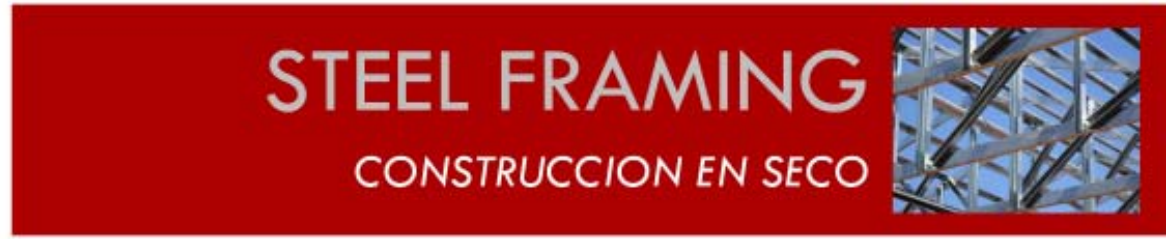


11. Tabla 1- Valores de transmitancia térmica estivales e invernales para la zona I (a y b), para muros y techos, en cada nivel de confort higrotérmico, según Norma IRAM 11605: 96.

talle del proceso de realización de cada conjunto y subconjunto funcional y ajuste definitivo del sistema; 5) una planilla individual de cómputo métrico de materiales y productos, según subconjunto funcional (fundaciones, estructuras, pisos, cerramientos laterales, cielorrasos, techo) y 6) una maqueta individual (física o digital).

La tercera y última etapa consistió en la síntesis grupal, en panel con formato de $1,00 \mathrm{~m} \times 0,70 \mathrm{~m}$, de la propuesta grupal y los programas arquitectónicos individuales desarrollados por los integrantes del grupo y de la propuesta tecnológica general (figura 10).

Se propuso que el panel viniera acompañado por una folletería (figura 11), diseñada con los mismos criterios gráficos y de contenidos que dicho panel, pero en formato manual, como para su eventual distribución durante una futura exposición de trabajos de los alumnos para planificar en la cátedra. El objetivo del panel-síntesis y su folletería adjunta fue que el equipo de alumnos pudiera comunicar los aspectos más importantes de las distintas propuestas de sus integrantes, lo que se constituyó en una situación "motivante", disparadora de su interés, por un lado hacia el tema tecnológico en sí, y por el otro, hacia la estrategia del marketing y comunicación de sus proyectos a posibles comitentes, etapa esta frecuentemente descuidada en el trayecto formativo del estudiante de arquitectura.

En cada una de las tres etapas del trabajo integrador desarrollado durante el cursado de la asignatura Construcciones II, se hizo hincapié en la consideración, por parte del alumnado, de aspectos muy generales referidos al ciclo de vida de los materiales y tecnologías empleados (metodología para evaluar el desempeño ambiental de un producto, proceso o actividad, a través de toda su existencia, desde la adquisición de materias primas hasta la disposición final).

\section{DISEÑO TECNOLÓGICO DE SOLU- CIONES ENERGETICAMENTE OPTI- MIZADAS}

Durante la segunda etapa del trabajo integrador desarrollado por los equipos, se abordó la cuestión del desempeño higrotérmico de la envolvente de los sistemas diseñados. Se hizo hincapié en que una manera eficiente de disminuir el consumo energético es utilizar una adecuada aislación térmica en techos y muros (envolvente constructiva = piel del edificio).

Los equipos de alumnos realizaron las verificaciones normativas vigentes, mediante las cuales constataron que utilizando las tecnologías de la construcción en seco de tipo "entramado", resulta sencillo incorporar las aislaciones, tanto para períodos invernales como estivales (reducción de pérdidas y ganancias térmicas, respectivamente).

Los valores máximos de transmitancia térmica (coeficiente " $K$ ") introducen exigencias relativas a la protección que deben brindar los cerramientos, a fin de garantizar condiciones ambientales de bienestar, con un razonable consumo energético para climatización, así como también evitar la aparición de fenómenos de condensación superficial de vapor de agua. Se establecen, en la Norma IRAM 11605: 1996, tres niveles de confort higrotérmico: NIVEL A: (recomendado o ecológico), NIVEL B (medio) y NIVEL C (mínimo). Para la zona bioambiental I, a la cual pertenece la región NEA, las normas establecen los valores de transmitancia que se exponen en la tabla 1, para muros y techos, según cuál sea el nivel de desempeño higrotérmico que se desee verificar y según se trate del período cálido o frío. ( Fig. 11).

A modo de ejemplo, en soluciones de cerramientos utilizando el sistema de construcción en seco tipo "steel frame", con caras exteriores tipo siding 
12. Valores de transmitancia térmica y nivel de confort higrotérmico para dos alternativas de muros de cerramiento con el sistema steel frame, una con relleno de EPS (izquierda) y otra con relleno de lana de vidrio (derecha)
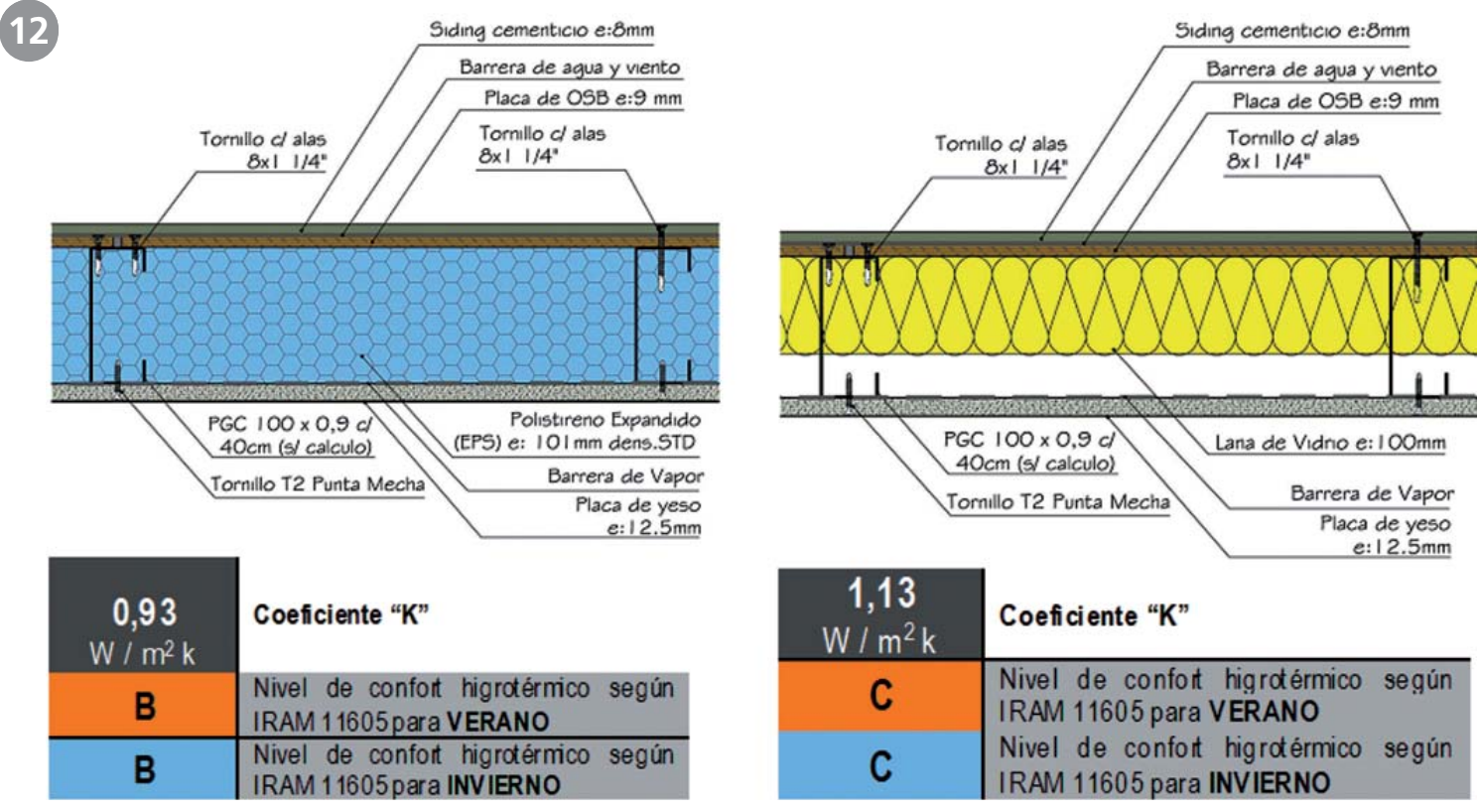

cementicio (tablillas de fibrocemento traslapadas) y almas rellenas con poliestireno expandido (EPS) o lana de vidrio (Fig. 12). Se logran valores de transmitancia de entre 0,93 y $1,13 \mathrm{~W} / \mathrm{m} 2 \mathrm{k}$, que corresponden a niveles de confort $B$ y $C$, respectivamente, mientras que una solución de muro de cerramiento habitual en nuestra región, como la representada por el muro de mampostería de ladrillos comunes de $0,30 \mathrm{~m}$. de espesor, revocado exterior e interiormente, tiene una transmitancia de $1,88 \mathrm{~W} / \mathrm{m}^{2} \mathrm{k}$, que representa un caso fuera de la categorización propuesta, por ser demasiado alto (ni siquiera se encuadra en el nivel C), a un espesor de cerramiento mucho mayor que las dos alternativas de construcción en seco ejemplificadas, en que el espesor total no supera los 0,20 m.

Por supuesto que aquí entran en consideración (y en torno a ello se han realizado debates y discusiones entre los equipos de alumnos) cuestiones relacionadas con el uso de materiales aislantes (EPS, lana de vidrio y otros), cuya inclusión lógicamente representa una inversión económica inicial mayor, pero que resultan amortizables en el tiempo, en plazos no superiores a cinco años, por los ahorros logrados en las facturas de electricidad del edificio, debidos a una menor demanda de equipos electromecánicos de climatización interior.

\section{LA CONSTRUCCIÓN SUSTENTABLE}

Teniendo en cuenta que "construir en forma sustentable" es una decisión estratégica para mejorar la calidad de vida y la economía local, se hizo hin- capié en que el proyecto de cada equipo de alumnos no involucrara una mayor inversión en tecnologías, sino la articulación de criterios de diseño arquitectónico con el uso eficiente de los recursos, intentando juntar estos elementos en pos de la optimización de la sustentabilidad en la construcción y funcionamiento de la vivienda (los edificios que se construyen bajo parámetros de sustentabilidad se caracterizan por ser energéticamente eficientes y tener un bajo impacto ambiental).

La construcción no convencional, y dentro de ella la construcción en seco del tipo "entramado" (steel frame y balloon frame), facilita la aplicación de pautas y criterios para una arquitectura sustentable. Entre las razones de ello, exploradas con los alumnos, pueden mencionarse las siguientes:

- Dado que se utilizan sistemas de montaje en seco, se facilita el desmontaje de componentes y su posterior inserción en otras construcciones (reutilización). - Al mismo tiempo, las labores de acoplamiento de las distintas partes generan menos residuos y un menor costo global que los sistemas de unión tipo húmedo. Hay una reducción drástica de los escombros y de los desperdicios o roturas de materiales en obra, dado que se diseña el edificio usando como "patrones modulares" las placas de los principales materiales utilizados.

- Las envolventes se diseñan y materializan mediante sistema multicapas, que otorgan la posibilidad de incorporar aislaciones térmicas en variados puntos, reduciendo el consumo de energía para calefacción y acondicionamiento. 
. surgió especialmente la necesidad de replanteo de las pautas particulares de seguimiento y correcciones... guías de trabajos prácticos, ya que estas opciones tecnológicas tienen particularidades..., que no tienen paralelo ni equivalente en otros sistemas de construcción en seco".

- Los costos ambientales resultan menores porque la construcción en seco utiliza elementos de fácil manejo y transporte. Se logra una economía en el transporte y sus emisiones ambientales nocivas, dado que hay menor incidencia de fletes para el traslado de materiales.

- Si el mantenimiento de los elementos y materiales utilizados no requiere operaciones de envergadura, la incidencia negativa en el ambiente se minimiza más inclusive. Por ejemplo: si las instalaciones sanitarias y eléctricas se proyectan inspeccionables y de fácil acceso, permiten optimizar las tareas de mantenimiento, reparación y desmontaje selectivo, posibilitando incluso la recuperación de conductos, líneas, mecanismos y aparatos, etc., para su ulterior reutilización o reciclado.

- Si se encara el diseño edilicio desde el punto de vista de la flexibilidad de uso de los espacios, ello debe de contribuir a la posibilidad de modificaciones en los locales (ampliaciones, etc.), sin que suponga grandes alteraciones en el esquema original. Frente a esta premisa, los sistemas de construcción en seco ofrecen amplias ventajas respecto de la construcción habitual, por la facilidad de desmontaje y la posibilidad de recuperación y reutilización de la mayor parte de los elementos y materiales.

- En el plano estructural, mediante los sistemas de "entramado" (steel y balloon frame) se logra un dimensionado estricto de las secciones, lo que minimiza el aporte de material y de elementos auxiliares y contribuye a la economía general del sistema.

La mayoría de estas cuestiones fueron trabajadas con los estudiantes durante los ciclos de cursado de Construcciones II - FAU - UNNE, como condicionantes y determinantes del proceso de diseño de los sistemas constructivos, lo que se verificó en los trabajos integradores de los equipos de alumnos.

\section{RESULTADOS Y PERSPECTIVAS}

Los puntos de innovación trabajados desde la cátedra han estado centrados en los contenidos, en las estrategias de enseñanza y de aprendizaje, en la articulación de la enseñanza con la investigación y sobre todo en la inclusión de aprendizajes centrados en el eje de la práctica. Las modificaciones introducidas se sintetizan en los siguientes puntos: (ALÍAS, PILAR Y VEDOYA, 2011): a) diversificación de los sistemas constructivos proyectados, en el campo de la construcción no convencional, incorporando criterios de sustentabilidad ambiental y eficiencia energética; b) diversificación de los programas arquitectónicos abordados, teniendo en cuenta problemáticas actuales y de impacto en el medio regional del nordeste argentino y c) incorporación de criterios de comunicación de las propuestas a posibles clientes e introducción al ámbito del marketing.

Las experiencias, específicamente las de las dos comisiones de los sistemas de entramado (balloon frame y steel frame), resultaron enriquecedoras: los alumnos pudieron madurar una idea más acabada de las diferencias y semejanzas, ventajas y desventajas, de las posibilidades tecnológicas que brinda actualmente la construcción en seco, profundizando en ellas, en tanto que los docentes pudimos verificar que los alumnos lograban establecer conexiones entre el desarrollo de su trabajo de diseño del sistema constructivo no convencional y los temas teóricos correlacionados que iban dándose como apoyatura, conforme avanzaba el desarrollo del cursado y del trabajo de integración. En estas comisiones de los sistemas de entramado surgió especialmente la necesidad de replanteo de las pautas particulares de seguimiento y correcciones, así como de los ítems de las guías de trabajos prácticos, ya que estas opciones tecnológicas tienen particularidades en cuanto a los componentes materiales, que no tienen paralelo ni equivalente en otros sistemas de construcción en seco. 
Asimismo, se verificó la integración de los conceptos sobresalientes de la arquitectura sustentable en los trabajos de diseño tecnológico-constructivo desarrollados por los equipos de alumnos, y la interiorización de dichos equipos en los aspectos normativos básicos referidos a las verificaciones de los principales parámetros higrotérmicos de las envolventes constructivas (transmitancia térmica y riesgo de condensaciones).

Los resultados obtenidos reflejan el nivel de conceptualización alcanzado por los estudiantes, tanto en relación con los contenidos inherentes a la tecnología constructiva no convencional, como con los incipientes criterios de sustentabilidad aplicados, referidos a hacer un menor uso de todos los recursos (materiales, económicos, etc.), sin sacrificar la calidad de vida del usuario, empleando materiales provenientes de fuentes menos contaminantes y generando la menor cantidad posible de desperdicios.

Con ello consideramos que el estudiante de $4 .^{\circ}$ año de Arquitectura logra integrar algunos criterios básicos de diseño ambientalmente consciente (racionalización constructiva, minimización de los costos energéticos y ambientales), lo que contribuye con el objetivo institucional de la FAU - UNNE de apuntar a que se vaya forjando gradualmente en el estudiante de arquitectura la conciencia de la necesidad de analizar el impacto que produce la arquitectura en la actualidad con una mayor amplitud, considerando toda la vida útil de los edificios, así como de difundir las características sobresalientes de la Arquitectura Sustentable (ALÍAS, MORÁN, PILAR, SCHUSTER Y VEDOYA; 2010).

La modalidad propuesta en Construcciones II, basada en la resolución del problema consistente en diseñar un sistema constructivo no convencional mediante la estrategia del taller y de una investigación paralela de los condicionantes y determinantes contextuales reales, se presenta, en este sentido, como un ámbito propicio para la deses- tructuración y para el ejercicio de prácticas alternativas de enseñanza y aprendizaje (ALÍAS; PILAR Y VEDOYA, 2011).

Tal como lo señala FERRY (1997), la formación es siempre un trabajo para sí mismo. En la medida en que el dispositivo pedagógico empleado otorgue justo valor y posibilidad de reflexión sobre sí mismo al alumno, y lo instrumente para el análisis de situaciones y episodios de la práctica y de la realidad del contexto, este alumno tendrá la oportunidad de avanzar en su trayecto de formación sobre sí mismo en distintos aspectos. Estamos convencidos de que, con la experiencia de Construcciones II, apuntamos en muchos aspectos a acompañar al alumno de $4 .^{\circ}$ año de la carrera de Arquitectura en un trayecto y proceso de este tipo. 


\section{BIBLIOGRAFIA}

GREVEN, H. A. y BALDAUF, A. S. F. (2007). Introdução à coordenação modular da construção no Brasil: Uma abordagem atualizada. Colección HABITARE. Asociación Nacional de Tecnología del Ambiente Construido. Porto Alegre - RS - Brasil.

ALÍAS, H. M.; MORAN, R.; PILAR, C. A.; SCHUSTER, A. y VEDOYA, D. E. (2010). "Criterios de sustentabilidad en el diseño de viviendas mediante sistemas constructivos no convencionales: experiencias en la enseñanza". Publicado en el CD de Ponencias en el marco del XXIX Encuentro y XIV Congreso Arquisur (Asociación de Facultades y Escuelas de Arquitectura Públicas del Mercosur). Departamento de Arquitectura y Urbanismo - Universidad Autónoma Juan Misael Saracho. Tarija, Bolivia.

ALÍAS, H. M.; PILAR, C. A. y VEDOYA, D. E. (2011). "La articulación teoría - práctica en la enseñanza de la construcción no convencional. La experiencia de la cátedra "Construcciones II" de la Facultad de Arquitectura de la UNNE", publicado en las Cuartas Jornadas de Comunicación de Experiencias Pedagógicas Innovadoras. ISBN 978-950-656-139-0. Programa de Formación Docente Continua. Secretaría General Académica. Universidad Nacional del Nordeste.

Instituto Argentino de Normalización y Certificación (IRAM). Normas Técnicas Argentinas: 11603: 1996; 11605: 1996. Buenos Aires, Argentina.

FERRY, G. (1997). Pedagogía de la Formación. Colección Formación de Formadores. Serie Los Documentos, Vol. 6. Facultad de Filosofía y Letras y Ediciones Novedades Educativas. Buenos Aires.

FINKELSTEIN, C. y LUCARELLI, E. (2003). La articulación teoría-práctica en un espacio curricular de formación en la profesión. Presentado en Congreso de Latinoamericano Educación Superior. UNSL San Luis. 